\title{
Review and Design of UWB Transmitter and Receiver
}

\author{
Rochan Banstola \\ Sikkim Manipal Institute of \\ Technology \\ SMIT, Rangpo Majitar \\ East Sikkim(INDIA)
}

\author{
Rabindranath Bera \\ Sikkim Manipal Institute of \\ Technology \\ SMIT, Rangpo Majitar \\ East Sikkim(INDIA)
}

\author{
Debasish Bhaskar \\ Sikkim Manipal Institute of \\ Technology \\ SMIT, Rangpo Majitar \\ East Sikkim(INDIA)
}

\begin{abstract}
Impulse radio communication systems and impulse radars both utilizes very short pulses in transmission that results in an ultra-wideband spectrum. This paper discusses the various techniques for transmission of the UWB impulses and also some receiver techniques. Also the design consideration for THSS-UWB transmitter using Matlab Simulink block is presented.
\end{abstract}

\section{General Terms}

Pulse Position Modulation, FCC's First Report and Order Cross Correlation.

\section{Keywords}

UWB, THSS, PPM

\section{INTRODUCTION}

The concept of Ultra-Wideband (UWB) application to radar and communications systems has been around since late 1950's[1].UWB technology is quickly gaining acceptance as a wireless technology with outstanding characteristics.UWB systems have been described using a variety of terms such as impulse radio (or radar), ultra wideband systems, time modulation systems, baseband (or pulse) systems and others.

As defined by the FCC's First Report and Order, UWB signals must have bandwidths of greater than $500 \mathrm{MHz}$ or a fractional bandwidth larger than 20 percent at all times of transmission [2]. Fractional bandwidth is a factor used to classify signals as narrowband, wideband, or ultra-wideband and is defined by the ratio of bandwidth at $-10 \mathrm{~dB}$ points to center frequency. Equation 1 shows this relationship.

$$
\begin{array}{r}
B f=\frac{B W}{f c} * 100 \% \\
B_{f}=\frac{f_{h}-f_{l}}{\left(\frac{f_{h}+f_{l}}{2}\right)} * 100 \%=\frac{2\left(f_{h}-f_{l}\right)}{\left(f_{h}+f_{l}\right)} * 100 \%
\end{array}
$$

Where $f_{h}$ and $f_{l}$ are highest and lowest cutoff frequencies (at the $-10 \mathrm{~dB}$ point) of UWB pulses spectrum respectively.

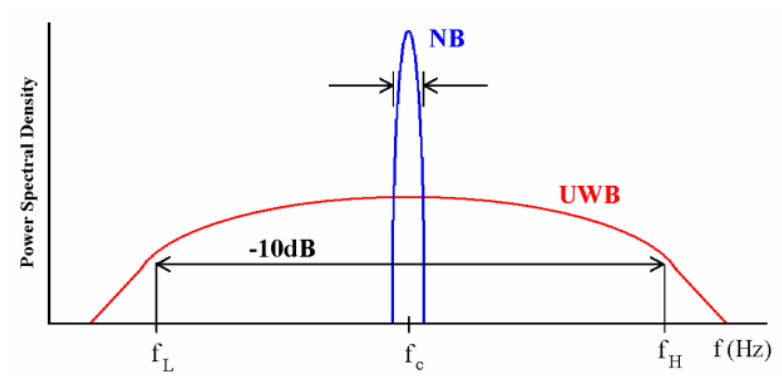

Fig 1: Power Spectral density at -10dB points

The recent approval of UWB technology by Federal Communications Commission (FCC) of the United States reserves the unlicensed frequency band between 3.1 and 10.6 $\mathrm{GHz}(7.5 \mathrm{GHz})$ for indoor UWB wireless communication systems. Ultra Wideband Technology have several useful applications like positioning, locating, penetrating through walls, wireless Ad Hoc Networks, tracking objects etc make this feature unique. Ultra Wideband (UWB) impulse radio proposed in [3] has a promising future.

This paper is organized as follows. In section II mathematical model of the signal pulse generation, modulation technique, and time hopping pulse position modulation is formulated. In section III and IV proposed design of UWB transmitter and receiver.

\section{MATHEMATICAL MODEL}

\subsection{Signal pulse generation}

A UWB signal can be anyone of a variety of wideband signals, such as Gaussian, chirp, wavelet, or Hermite-based short-duration pulses. In the FCC specification, the allowed PSD mask for UWB signal is a rectangular shape. It is obvious that the Gaussian shaped pulses do not match with this rule perfectly. Therefore, the Raised Cosine pulse is introduced to provide a better matching with the FCC mask. The Raised Cosine pulse that is utilized in this system is described by equation 2 and the pulse is shown in fig2. While most UWB providers use some version of this waveform, there are several different approaches to implementing UWB systems [4].

$$
V(t)=e^{-\frac{t^{2}}{2}} \cdot \cos (5 t)
$$




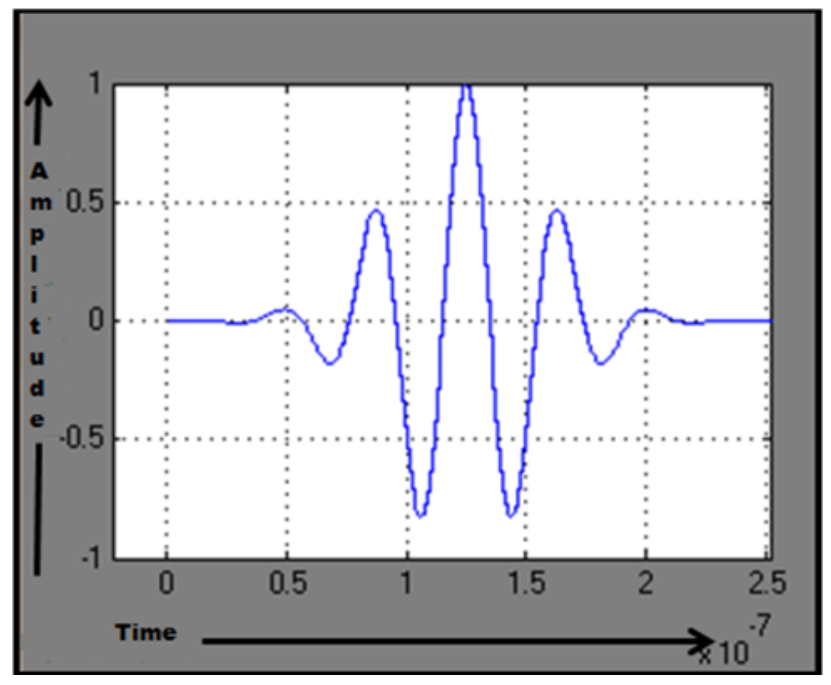

Fig 2: UWB Pulse

\subsection{Modulation techniques}

The most common impulse radio concepts are based on Pulse Position Modulation. These techniques use time shift of regularly timed pulses, as it is shown at fig.3 [5] for two modulation states. Any two distinct modulation states can encode binary information. More than two states can be used. Generally, it can be defined as $M$ time shifts for $M$ modulation states. Analytically, the signal can be represented as

$$
S_{i}(t)=p\left(t-\tau_{i}\right)
$$

Where $\mathrm{p}(\mathrm{t})$ is waveform at unmodulated nominal position, $\tau_{i}$ is time shift for $\mathrm{i}^{\text {th }}$ modulation state.

The best theoretical performance of PPM in AWGN channel can be achieved with non overlapping orthogonal pulses. However, autocorrelation functions of many usable pulses have both positive and negative values. This allows as achieving a better BER performance than only that for time orthogonal pulses, in the case of two modulation states. Because of large spectral peaks of PPM streams, pulses should not be evenly spaced in time, but they should be spaced at pseudorandom intervals. This technique is called Time Hopping. TH is in principle pseudorandom M-ary PPM, but not for data modulation. TH is often used in TH-PPM combination. It creates a noise like signal and allows multiple accesses. PPM can also be used in combination with Direct Sequence technique. PPM requires very precise pulse timing.

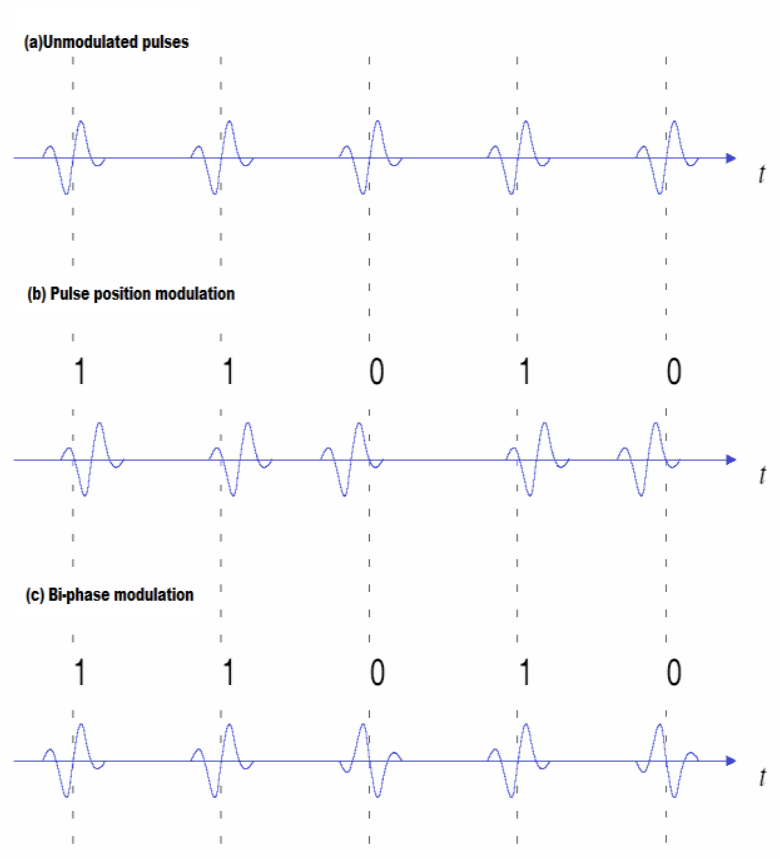

Fig 3(a) Unmodulated waves (b) PPM modulated wave (c) Bi-phase modulated wave.

\subsection{Time hopping pulse position modulation} The time hopping M-ary PPM system model for kth user is given by

$$
S^{k}(t)=A_{\frac{d}{N}}^{k} p\left(t-j T_{f}-c_{j}^{(k)} T_{c}-\delta d_{\frac{j}{N}}^{(k)}\right)
$$

Where $\mathrm{A}(\mathrm{k})$ is the signal amplitude, $\mathrm{p}(\mathrm{t})$ represents the derivative of Gaussian pulse with pulse width $\mathrm{Tp}$ and $\mathrm{Tf}$ being the frame time. The frame is divided into $\mathrm{N}$ time slots with duration Tc. The pulse shift pattern cj(k), $0 \leq \operatorname{cj}(\mathrm{k}) \leq \mathrm{N}$ also called the time hopping sequence for kth source and it is pseudo random with period Tc, this additional shift avoids the catastrophic collision due to multiple access interference (MAI). The time hopping spread spectrum is shown in fig4. [6]

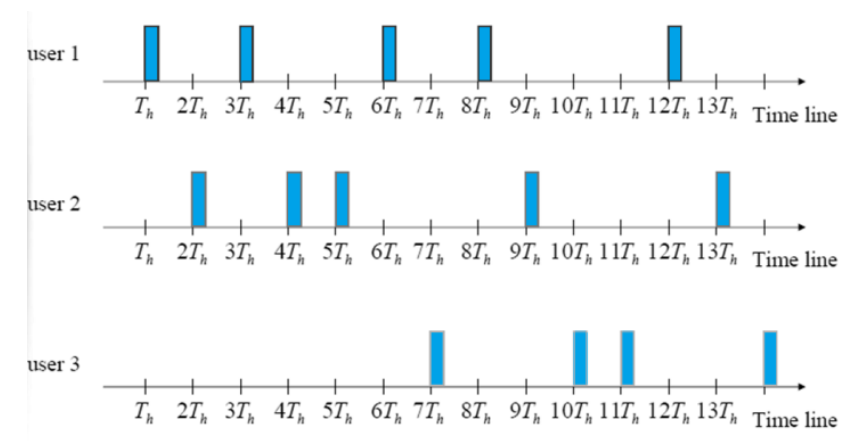

Fig 4: Time hopping spread spectrum. 


\section{UWB TRANSMITTER}

The schematic block diagram of UWB transmitter design is shown in the figure. In this model, typical indoor multiuser communication scenario and TH-PPM as the signaling format is considered. The transmission scheme from different user is assumed to be asynchronous. Each user is assigned a pseudorandom time hopping sequence to avoid catastrophic collision with other users.

TH-UWB first generates a typical time shifting or time hopped with pulse position modulation pulse. This pulse is use to generate the UWB signal. A typical UWB signal generated by the kth transmitter (or kth user) is shown in equation 4 .

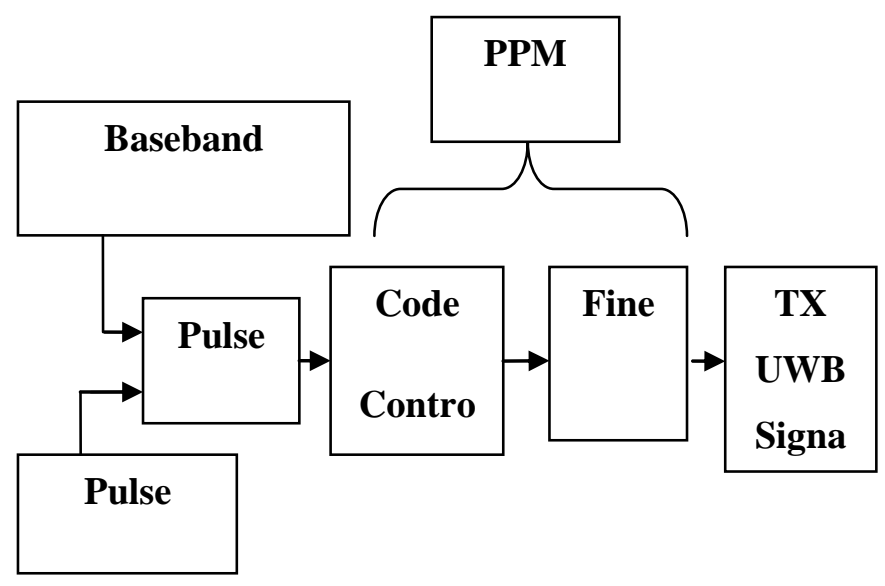

Fig 5: Block diagram of UWB transmitter.

The block diagram of the designed UWB transmitter is shown in Fig5. Here the binary information stream is passed to the 'front end', which is the part of the transmitter which the system is concerned about. For higher modulation schemes the binary information should be mapped from bits to symbols, with each symbol representing multiple bits. These symbols are then mapped to an analog pulse shape. Pulse shapes are generated by the pulse generator. For PPM the timing must be even more precise, usually less than one pulse width.

\section{UWB RECEIVER}

The analysis of the detection and reception of the signals are based on AWGN (additive white Gaussian noise) in this model. The conventional pulse-detection techniques used were Energy detectors and Classical matched filters (CMF). Most of the UWB receivers use one of these techniques for data demodulation. Energy detectors are simple, non-coherent receivers that detect the energy of a signal \& thus compare it with threshold level to demodulate data bits [7].

The receiver basically measures the energy of the received signal $r(t)$ in two parts and selects the symbol corresponding to the maximum energy. However the optimal receiver is the correlation based receiver [6] described in figure5. The principle consists of locally generating a template waveform $v(t)$ which is multiplied with the received signal $r(t)$. The PPM demodulator computes the sign of the correlator output accumulated over the $\mathrm{N}_{\mathrm{f}}$ frames of one symbol.

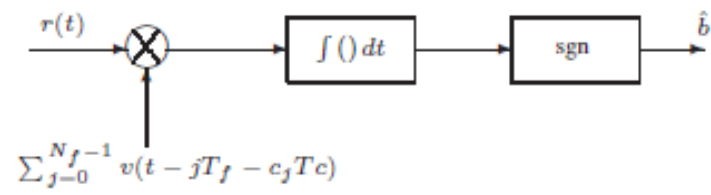

Fig 6: Optimal UWB receiver

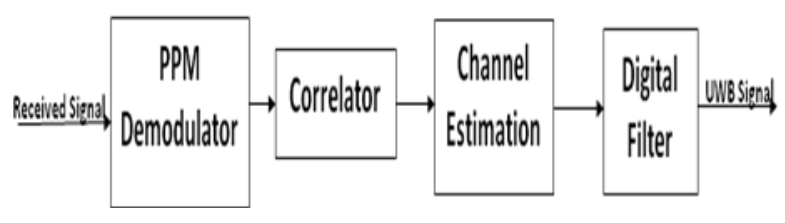

Fig 7: Block diagram of UWB receiver.

The receiver model designed for the simulation is depicted in Fig6. Here the received signal through the AWGN channel is demodulated using the PPM demodulator. The fine positioned delay $\delta d^{(k)}$ is first extracted and then accordingly compensated by its respective positional index. This pulse position demodulation is done by compensating this position delay of each pulse.As the delay is compensated then a cross correlation is done with the reference UWB pulsed Morlet.

\section{SIMULATION AND RESULTS}

For the simulation of the complete uwb system, tool utilized is Matlab (2012). The designed is based on the Simulink block of Matlab. The blocks of both the transmitter and receiver are shown in figure 5 and figure 7 respectively. The transmitter signal that has been corrupted by the thermal noise in the AWGN channel is also shown in figure 8 .

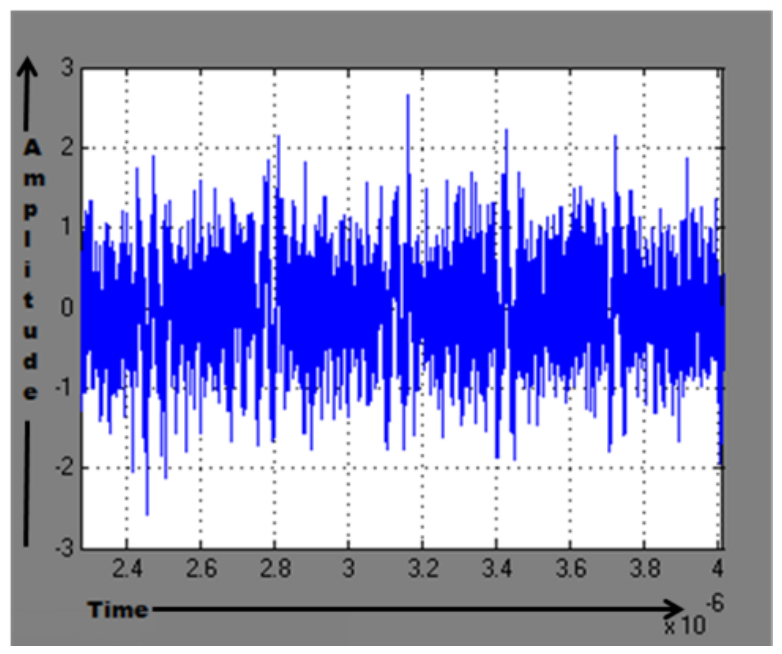

Fig 8: AWGN channel out. 
The output of the transmitter is shown in figure 9. The received waveform shown is delayed by few samples. The zoom in version of same is shown in figure 10.The pulse is distorted due the channel effects. Moreover the channel used in this model is AWGN, there is always chance of signal distortion due to the thermal noise.

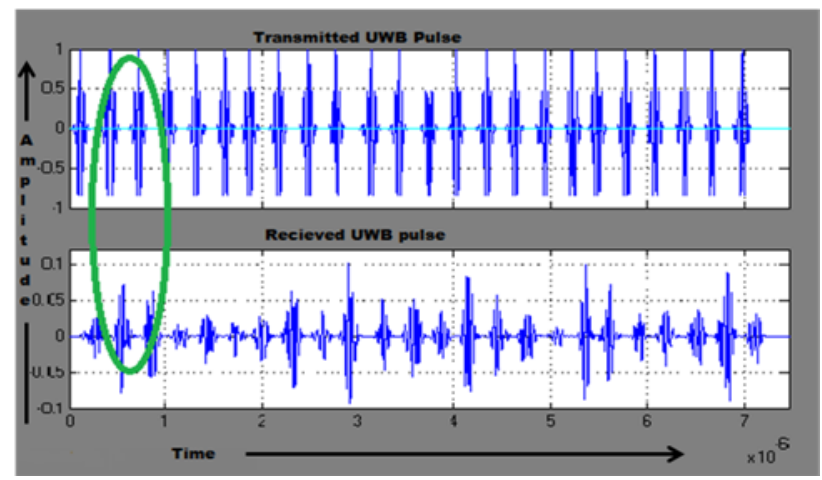

Fig 9: Transmitted and Received Signal.

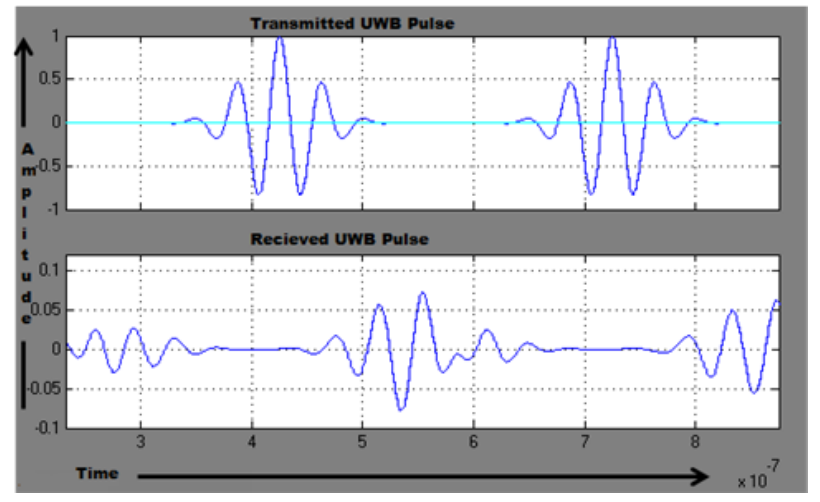

Fig 10: Zoom in version of Transmitted and Received Signal

Table 1. Application wise frequency band with user limitation

\begin{tabular}{|l|l|c|}
\hline Class/Application & $\begin{array}{c}\text { Frequency Band } \\
\text { for Operation }\end{array}$ & User limitation \\
\hline $\begin{array}{c}\text { Communication } \\
\text { and Measurement } \\
\text { Systems }\end{array}$ & 3.1 to $10.6 \mathrm{GHz}$ & No \\
\hline $\begin{array}{c}\text { Imaging: Ground } \\
\text { Penetration, } \\
\text { Radar, Wall, } \\
\text { Medical Imaging }\end{array}$ & $\begin{array}{c}<960 \mathrm{MHz} \text { or } 3.1 \\
\text { to } 10.6 \mathrm{GHz}\end{array}$ & Yes \\
\hline $\begin{array}{l}\text { Imaging: Through } \\
\text { Wall }\end{array}$ & $\begin{array}{l}<960 \mathrm{MHz} \text { or } 1.99 \\
\text { to } 10.6 \mathrm{GHz}\end{array}$ & Yes \\
\hline $\begin{array}{l}\text { Imaging: } \\
\text { Surveillance }\end{array}$ & $\begin{array}{l}1.9 \mathrm{MHz} \text { to } 10.6 \\
\mathrm{GHz}\end{array}$ \\
\hline
\end{tabular}

The above table shows the frequency band of operation in Ultra Wide Band range for different application. It also shows the limitation of the users in the given band and the application. The proposed model designed here can be utilized in the given application by changing the bandwidth of the system in the Simulink model. However, there always lies the designing complexity when the model is upgraded towards the wider bandwidth.

\section{CONCLUSION}

The uwb system purposed by different researchers has been reviewed and a complete uwb system using Matlab Simulink has been designed. It could also be seen that the optimal receiver for such kind of system is necessarily be a correlation receiver.

Since here the pulse used is of very short duration typically 0.5 nano seconds, this system can used for solving the wall penetration problem in the fields like fire fighting areas. Further deployment of UWB technology can highly enhanced the future wireless technology

\section{REFERENCES}

[1] Testing and Research on Interference to GPS from UWB Transmitters Ming Luo, Dennis Akos, Michael Koenig, , Guttorm Opshaug, Sam Pullen, and Per Enge Stanford University Bob Erlandson Collins Rockwell Corp. / RTCA Sally Frodge U.S. Department Of Transportation.

[2] Revision of part 15 of Commission Rules regarding Ultra-Wideband Transmission systems, Source:http://www.fcc.gov/Bureaus/Engineering_Techno logy/Orders/2002/fcc02048.pdf.

[3] Scholtz R.A., "Multiple access with time-hopping impulse modulation," MILCOM '93 Proceedings, vol. 2, pp. $447-450,1993$.

[4] Time Domain's Ultra Wideband (UWB) Definition and Advantages 320-0294B June 2012.

[5] Uwb Pulse Modulation Methods Petr Bezucha IREE CAS Prague, Chaberská 570

[6] UWB: Impulse Radio for Radar and Wireless Communications(Virginia tech)

[7] UWB Communication Receiver: Review and Design Considerations Rahul Malhotra* et al. / (IJAEST) International Journal Of Advanced Engineering Sciences And Technologies Vol No. 8, Issue No. 2, 197 - 202

[8] Security Embedding on UWB-IR Physical Layer. Globecom 2012-Communication and Information System Security Symposium. 